\title{
The alternative sigma factor RpoQ regulates colony morphology, biofilm formation and motility in the fish pathogen Aliivibrio salmonicida
}

\author{
Miriam Khider, Nils Peder Willassen and Hilde Hansen ${ }^{*}$ (D)
}

\begin{abstract}
Background: Quorum sensing (QS) is a cell-to cell communication system that bacteria use to synchronize activities as a group. LitR, the master regulator of QS in Aliivibrio salmonicida, was recently shown to regulate activities such as motility, rugosity and biofilm formation in a temperature dependent manner. LitR was also found to be a positive regulator of $r p o Q$. RpoQ is an alternative sigma factor belonging to the sigma -70 family. Alternative sigma factors direct gene transcription in response to environmental signals. In this work we have studied the role of RpoQ in biofilm formation, colony morphology and motility of A. salmonicida LFI1238.

Results: The rpoQ gene in A. salmonicida LFI1238 was deleted using allelic exchange. We found that RpoQ is a strong repressor of rugose colony morphology and biofilm formation, and that it controls motility of the bacteria. We also show that overexpression of $r p o Q$ in a $\Delta$ litR mutant of $A$. salmonicida disrupts the biofilm produced by the $\Delta$ litR mutant and decreases its motility, whereas $r p o Q$ overexpression in the wild-type completely eliminates the motility.

Conclusion: The present work demonstrates that the RpoQ sigma factor is a novel regulatory component involved in modulating motility, colony morphology and biofilm formation in the fish pathogen A. salmonicida. The findings also confirm that RpoQ functions downstream of the QS master regulator LitR. However further studies are needed to elucidate how LitR and RpoQ work together in controlling phenotypes related to QS in A. salmonicida.
\end{abstract}

Keywords: Aliivibrio salmonicida, Sigma factors, RpoQ, Temperature, Quorum sensing, Motility, Biofilm, Overexpression

\section{Background}

Aliivibrio salmonicida belongs to the Vibrionaceae family, which is widely distributed in the environment, mainly in the aquatic habits. Members of this family may exist in symbiotic or pathogenic relations with their hosts [1]. According to current taxonomy, A. salmonicida belongs to the Aliivibrio genus together with its three most closely related species Aliivibro logei, Aliivibrio wodanis and Aliivibrio fischeri [2].

A. salmonicida causes cold water vibriosis or Hitra disease in farmed Atlantic salmon (Salmo salar L), Atlantic cod (Gadus morhua) and rainbow trout (Oncorhynchus

\footnotetext{
* Correspondence: hilde.hansen@uit.no

Norwegian Structural Biology Center and the Department of Chemistry, Faculty of Science and Technology, UiT-The Arctic University of Norway,
} $\mathrm{N}-9037$ Tromsø, Norway

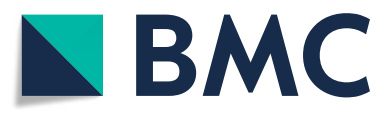

(อ) The Author(s). 2018 Open Access This article is distributed under the terms of the Creative Commons Attribution 4.0 International License (http://creativecommons.org/licenses/by/4.0/), which permits unrestricted use, distribution, and reproduction in any medium, provided you give appropriate credit to the original author(s) and the source, provide a link to the Creative Commons license, and indicate if changes were made. The Creative Commons Public Domain Dedication waiver (http://creativecommons.org/publicdomain/zero/1.0/) applies to the data made available in this article, unless otherwise stated. and winter seasons when the seawater temperature is below $12^{\circ} \mathrm{C}$. A salmonicida is a gram-negative psychrophilic bacterium with a rod shape and nine polar flagella for motility and colonization [3-5].

Members of the Vibrionaceae family use quorum sensing (QS) for cell-to-cell communication to regulate gene expression in response to cell density by secretion and sensing of extracellular signals called auto-inducers (AIs). As the bacterial population density increases, AIs accumulate in the environment. When the AI concentration increases above a certain threshold, the bacteria detect this and modulate gene expression [6,7]. N-acyl homoserine lactones (AHLs) are the major class of AIs in gram-negative bacteria, and were first described in $A$. fischeri $[8,9]$ and Vibrio harveyi $[10]$. The QS systems in 
A. fischeri control properties such as motility, squid colonization and bioluminescence [11-13]. A. fischeri has two AHL based systems, LuxI/LuxR and AinS/AinR, which are primarily responsible for regulating bioluminescence and colonization factors [14]. In addition to the LuxI/LuxR and AinS/AinR systems, A. fischeri has the LuxS/LuxPQ QS system [14, 15]. LuxI is responsible for the synthesis of the autoinducer N-3-(oxo-hexanoyl)homoserine lactone (3-oxo-C6-HSL) which binds the cytoplasmic receptor LuxR. LuxR then functions as a transcription activator for the luciferase $\operatorname{luxICDABE}$ operon [16]. LuxS and AinS synthesize signal molecules which are sensed by LuxPQ and AinR, respectively. The two signal systems work in parallel and convey the signal responses to LuxU-LuxO. At low cell density when AIs are not produced, LuxPQ and AinR act as kinases and relay phosphates to LuxU, which in turns phosphorylates LuxO. Phosphorylated LuxO activates the transcription of $q r r$ which binds and destabilizes the mRNA of the master QS regulator LitR [12, 15, 17]. At high cell density, the AI produced by AinS (C8-HSL) accumulates in the environment and results in dephosphorylation of LuxO. When LuxO is dephosphorylated, the qrr level decreases and allows LitR translation. In turn, LitR activates the transcription of $l u x R$ which contributes to bioluminescence $[12,13]$.

A. salmonicida has three QS systems similar to those in A. fischeri: LuxS/LuxPQ, LuxI/LuxR and AinS/AinR [18]. LuxI is responsible for the synthesis of a total of seven AHLs, while AinS synthesizes only one AHL. This AHL diversity may suggest a complex sensing system which allows more fine-tuned responses to changes in the environment [19]. A. salmonicida does not produce bioluminescence per se [20], but regulates activities such as virulence, motility, colony morphology, adhesion, and biofilm formation by QS in a temperature dependent manner [21, 22].

Sigma factors are essential dissociable subunits of prokaryotic RNA polymerase that control promoter recognition and transcription initiation [23, 24]. Primary sigma factors (RpoD, $\sigma^{70}$ family) direct transcription from the promoters of genes required for basic cellular functions. In addition to the primary sigma factors, bacteria have a variable number of alternative sigma factors whose activities increase in response to certain environmental conditions or stress [25].

Several alternative sigma factors have been identified or predicted in vibrios and aliivibrios [26], and recently a divergent copy of a putative RpoS-like sigma factor was identified in A. fischeri and named RpoQ due to its activation by the AinS/AinR QS system [27]. RpoQ was later found to regulate bioluminescence, motility and chitinase activity in $A$. fischeri through LuxO via LitR [28]. Pfam analysis of RpoQ identified four conserved domains $\left(\sigma^{70}\right.$ regions) where all were significant except for region 3. Phylogenetic analysis further revealed that region 3 in RpoQ is clearly divergent from the corresponding region in RpoD and RpoS [27, 28]. This less conserved region 3 is involved in binding the core RNA polymerase and recognition of the extended -10 promoter [29]. An RpoS-like sigma factor (RpoX) lacking region 3 has been described in Vibrio alginolyticus, and shown to be involved in biofilm formation and stress responses [30].

A. salmonicida strain LFI1238 encodes an rpoQ homolog (VSAL_IIO319) similar to the one in A. fischeri $[18,28]$. In a previous study we analyzed the transcriptomes of an A. salmonicida $\Delta$ litR mutant and the isogenic wild-type strain LFI1238. The rpoQ gene was found to be downregulated in the $\Delta l i t R$ mutant [31] suggesting that LitR is a positive regulator of $r p o Q$ in $A$. salmonicida. In the work presented here we have studied the impact of this putative RpoS-like sigma factor in A. salmonicida with regard to different phenotypic traits such as biofilm formation, motility and colony morphology.

\section{Methods}

Bacterial strains, plasmids and culture conditions

Bacterial cells and plasmids used in this study are listed in Table 1 . The wild-type A. salmonicida LFI1238 and the constructed mutants were grown from frozen glycerol stocks on blood agar base no. 2 (Oxiod, Cambridge, United Kingdom) with a final concentration of $2.5 \% \mathrm{NaCl}$ (wt $/ \mathrm{vol}$ ) and $5 \%$ bovine blood (BA2.5) or on Luria-Bertani agar (Difco, BD Diagnostics, Sparks, MD) with a final concentration of $2.5 \% \mathrm{NaCl}$ (wt/vol) (LA2.5). The primary cultures ( $2 \mathrm{ml}$ ) of $A$. salmonicida and the constructed mutants were grown from single colonies in LB2.5 at $12^{\circ} \mathrm{C}$ and $220 \mathrm{rpm}$ for $48 \mathrm{~h}$. Secondary cultures were made by diluting the primary cultures 1:20 in LB2.5 and incubated for additional $24 \mathrm{~h}$, unless otherwise indicated.

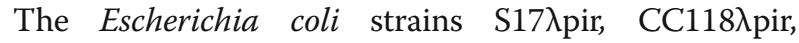
JM109, PIR2, DH5 $\alpha \lambda$ pir and DH5 $\alpha$ were cultivated in LA or LB with $1 \%$ (wt/vol) $\mathrm{NaCl}$ (LA1 and LB1 respectively) and incubated at $37^{\circ} \mathrm{C}$. The suicide plasmids pDM4 (GenBank: KC795686.1) and pNQ705 (GenBank: KC795685.1) were propagated in S17 $\lambda$ pir cells. The TA plasmid vector pGEM-T was propagated in JM109 and DH5 $\alpha$ cells. The conjugation helper pEVS104 plasmid was propagated in the E. coli helper strain CC118 $\lambda$ pir [32]. The pTM214 and pVSV102 (GFP) expression plasmids were propagated in the donor strains PIR2 and DH5 $\alpha \lambda$ pir, respectively $[32,33]$. For selection of $E$. coli transformants, chloramphenicol (final concentration $25 \mu \mathrm{g} / \mathrm{ml}$ ) or ampicillin (final concentration $100 \mu \mathrm{g} / \mathrm{ml}$ ) was added to the medium. The potential A. salmonicida transconjugants were selected either on BA2.5 or LA2.5 supplemented with $2 \mu \mathrm{g} / \mathrm{ml}$ of chloramphenicol or $150 \mu \mathrm{g} / \mathrm{ml}$ of kanamycin. 
Table 1 Bacterial strains and plasmids used in this study

\begin{tabular}{|c|c|c|}
\hline Bacterial strains or plasmids & Description & Source \\
\hline \multicolumn{3}{|l|}{ A. salmonicida } \\
\hline LFI1238 & Wild-type, isolated from Atlantic cod & [18] \\
\hline$\Delta l i t R$ & LFI1238 containing an in-frame deletion in litR & [22] \\
\hline$\Delta r p o Q$ & LFl1238 containing an in-frame deletion in rpoQ & This study \\
\hline$\Delta r p o Q_{c}$ & $\Delta r p o Q$ strain complemented with wild-type copy of the rpoQ gene, $\mathrm{Cm}^{r}$ & This study \\
\hline$\Delta l i t R-r p o Q^{-}$ & $\Delta l i t R$ stain with an insertional disruption in $r p o Q, \mathrm{Cm}^{r}$ & This study \\
\hline LFI1238-pVSV102 & LFI1238 carrying pVSV102, $\mathrm{Kn}^{\mathrm{r}}$ & This study \\
\hline AlitR-pVSV102 & $\Delta$ litR carrying pVSV102, $\mathrm{Kn}^{\mathrm{r}}$ & This study \\
\hline$\Delta r p o Q-p V S V 102$ & ArpoQ carrying pVSV102, $\mathrm{Kn}^{r}$ & This study \\
\hline LF11238-pTM214 & LFI1238 carrying pTM214, $\mathrm{Cm}^{\mathrm{r}}$ & This study \\
\hline LFI1238-Ptrc-rpoQ & LFI1238 carrying pTM214-rpoQ, $\mathrm{Cm}^{r}$ & This study \\
\hline$\Delta$ litR-pTM214 & $\Delta$ litR carrying pTM214, $\mathrm{Cm}^{\mathrm{r}}$ & This study \\
\hline$\Delta$ litR-Ptrc-rpoQ & $\Delta$ litR carrying pTM214-rpoQ, $\mathrm{Cm}^{r}$ & This study \\
\hline \multicolumn{3}{|l|}{ E. coli } \\
\hline S17גpir & Donor strain for conjugation & [65] \\
\hline JM109 & Strain for subcloning pGEM-T constructs & [66] \\
\hline $\mathrm{DH} 5 \mathrm{a}$ & Strain for cloning & Thermo Fisher \\
\hline C118入pir & Helper strain containing pEVS104 & [32] \\
\hline DH5aגpir & Donor strain for conjugation harboring pVSV102 & [32] \\
\hline PIR2 & Donor strain for conjugation harboring pTM214 & [33] \\
\hline \multicolumn{3}{|l|}{ Plasmids } \\
\hline pDM4 & Suicide vector with an R6K origin, sacBR and $\mathrm{Cm}^{r}$ & [35] \\
\hline pNQ705 & Suicide vector with an R6K origin, $\mathrm{Cm}^{r}$ & [35] \\
\hline pDM4- $\Delta r p o Q$ & pDM4 containing a fragment of rpoQ harboring an internal deletion & This study \\
\hline pNQ705-rpoQ $c$ & pNQ705 containing a full length rpoQ and flanking sequences & This study \\
\hline pNQ705-rpoQ ${ }^{-}$ & pNQ705 containing an internal $304 \mathrm{bp}$ fragment of rpoQ & This study \\
\hline pTM214 & pVSV105, Ptrc-mCherry, Cm ${ }^{r}$ & [33] \\
\hline pVSV102 & pES213, constitutive GFP, $\mathrm{Kn}^{\mathrm{r}}$ & [67] \\
\hline pEVS104 & Helper plasmid, R6K origin, RP4, oriT, trb, tra and $\mathrm{Kn}^{\mathrm{r}}$ & [32] \\
\hline pTM214-rpoQ & pVSV105, Ptrc-rpoQ (a full length rpoQ copy), $\mathrm{Cm}^{\mathrm{r}}$ & This study \\
\hline PGEM-T & TA cloning vector, white/blue screening, Amp ${ }^{r}$ & Promega \\
\hline
\end{tabular}

A seawater-based medium (SWT) was used for biofilm and morphology assays. The medium contains $5 \mathrm{~g} / \mathrm{L}$ of bacto peptone (BD), $3 \mathrm{~g} / \mathrm{L}$ of yeast extract (Sigma) and $28 \mathrm{~g}$ of a synthetic sea salt (Instant Ocean, Aquarium Systems) per liter. The SWT medium was solidified with $1.5 \%$ (wt/vol) agar (Fluka).

All biological assays were carried out in triplicate.

\section{DNA extraction, PCR and DNA sequencing}

DNA extraction, recombinant DNA techniques and transformations were performed according to standard protocols [34]. Restriction digestion, ligation, genomic DNA extraction and plasmid purification were performed as recommended by the manufacturers (NEB
Biolabs, Sigma and Promega). PCR was performed using Phusion polymerase (NEB) or Taq polymerase master mix (WVR). DNA sequencing was performed using Big Dye (Applied Biosystems) with custom made primers synthesized by Sigma. The primers used for PCR and sequencing are listed in Table 2.

Construction of $A$. salmonicida LFI1238 $\Delta r p o Q$ mutant and the complementary strain

The rpoQ gene (VSAL_IIO319) was deleted in A. salmonicida by allelic exchange as previously described [22] In brief, the pDM4- $\triangle r p o Q$ was constructed by fusion of two PCR products amplified from sequences downstream and upstream rpoQ in the genomic DNA of $A$. 
Table 2 The primers used in this study

\begin{tabular}{lll}
\hline Primers & Sequence (5-3') & Source \\
\hline RpoQ-A fwd & AATAACTCGAGCAAACGAATGACATGCAGACA & This study \\
RpoQ-B rev & ATCAATGCTGTTCTTGGTCTTC & This study \\
RpoQ-C fwd & AGAAACAGCATTGATCTAGGCCAAGATCTTCAA & This study \\
RpoQ-D rev & TATATACTAGTCGATCTCATTATCTTCGTAATACA & This study \\
RpoQ-G fwd & AGTTCAGGTGATCGTGTTA & This study \\
RpoQ-H rev & GATTTGGGTATTGGTAACT & This study \\
RpoQ-E fwd & CTCGAGAACAGCATTGATGCTTACTCA & This study \\
RpoQ-F rev & ACTAGTATCCACCATACCGCGTAA & This study \\
pTM214-rpoQ fwd & TCGAGCTCAGAGGAGAAATTAAGCATGTTGAATATAGAATGTTCA & This study \\
pTM214-rpoQ rev & AGGTCGACCTAATTTAAAGCATTTCTAAA & This study \\
pNQ-fwd & TAACGGCAAAAGCACCGCCGGACATCA & Milton, D. \\
pNQ-rev & TGTACACCTAACACTCGCCTATTGT & Milton, D. \\
\hline
\end{tabular}

salmonicida LFI1238. The RpoQ-A and RpoQ-B primers were used to amplify the region upstream rpoQ (558 bp), and RpoQ-C and RpoQ-D primers for amplification of the region downstream rpoQ (729 bp). The downstream region contained the last $40 \mathrm{C}$-terminal codons of the $r p o Q$ open reading frame. Primers RpoQ-B and RpoQ-C contain complementary sequences that enable fusion of the upstream and downstream PCR products by a second overlap-extension PCR. This fusion of the two PCR products results in removing 254 codons (including the start codon) from the rpoQ open reading frame. A'overhangs were added to the PCR product and ligated into pGEM-T, and transformed into E. coli JM109 competent cells. The insert (PCR overlap product) was digested from the pGEM-T plasmid using SpeI and XhoI, as restriction sites are included in RpoQ-A and RpoQ-D primers respectively. The digested overlap PCR product was then ligated into the corresponding restriction sites of the suicide vector pDM4 before being transformed directly to $E$. coli S17 $\lambda$ pir cells. The resulting plasmid is named pDM4- $\Delta r p o Q$.

The complementary strain $\Delta r p o Q_{c}$ was constructed by insertion of a full-length copy of the wild-type rpoQ gene into the original locus of the $\Delta r p o Q$. The complete gene and flanking regions was amplified by PCR using RpoQ-A and RpoQ-D primers, digested as above, and ligated into the SpeI and XhoI restriction sites of the pNQ705. The resulting plasmid is named $\mathrm{pNQ705-rpo} Q_{c}$.

The pDM4- $\Delta r p o Q$ was transferred to A. salmonicida LFI1238, while the pNQ705-rpo $Q_{c}$ construct was transferred to the $\triangle r p o Q$ mutant by bacterial conjugation mainly as described elsewhere [22, 35]. Briefly, donor

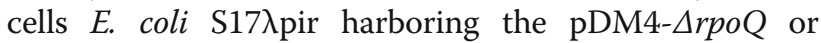
pNQ705-rpo $Q_{c}$ were mated with their respective recipient cells (A. salmonicida wild-type or the $\triangle r p o Q \mathrm{mu}$ tant), at a 1:1 ratio. The donor cells were grown to mid-exponential phase to $\mathrm{OD}_{600}$ (optical density) of 0.7 and the recipient to an early stationary phase $\left(\mathrm{OD}_{600}\right.$ 1.2) before they were harvested by centrifugation and washed twice in LB1 medium. The washed bacterial pellets were mixed and spotted onto BA2.5 agar plates. The plates were incubated at $20^{\circ} \mathrm{C}$ for $6 \mathrm{~h}$ followed by an additional incubation for $17 \mathrm{~h}$ at $12^{\circ} \mathrm{C}$. The spotted cells were suspended in $2 \mathrm{ml} \mathrm{LB} 2.5$ and incubated overnight at $12^{\circ} \mathrm{C}$ with agitation at $220 \mathrm{rpm}$. Potential transconjugants were selected after 5 days on BA2.5 supplemented with chloramphenicol. To complete the allelic exchange needed to generate the $\triangle r p o Q$ mutant, transconjugants (A. salmonicida-pDM4- $\Delta r p o Q)$, were streaked onto LA2.5 plate supplemented with $5 \%$ sucrose. Cells that are able to grow after the sucrose selection were selected based on the sensitivity to chloramphenicol. Chloramphenicol-sensitive cells were analyzed for deletion by PCR and verified by sequencing.

\section{Construction of the double mutant $A$. salmonicida $\Delta$ litR- rpoQ ${ }^{-}$}

Construction of A. salmonicida LFI1238 containing a litR in-frame deletion ( $\Delta l i t R)$ is described elsewhere [22]. The double mutant $\triangle$ litR-rpoQ $Q^{-}$(Table 1) was constructed mainly as described by others [35]. Briefly, the pNQ705-rpo ${ }^{-}$plasmid was constructed by cloning a (304 bp) PCR product amplified from an internal part of the $r p o Q$ gene using the forward and reverse primer pair RpoQ-E and RpoQ-F (Table 2). The restrictions enzyme sites SpeI and XhoI were added to the 5' end of the forward (RpoQ-E) and reverse (RpoQ-F) primers respectively in order to ligate the digested PCR product into the pNQ705 suicide plasmid. Hence, both the pNQ705 plasmid and the amplified PCR product were digested with SpeI and XhoI and ligated using T4 DNA ligase. The ligated construct (pNQ705-rpo ${ }^{-}$) was transformed into E. coli S17 pir. Next pNQ705-rpoQ was transferred to the $\Delta l i t R$ mutant by bacterial conjugation 
as described above. The resulting double mutant strain was named $\triangle$ litR-rpoQ $Q^{-}$.

\section{Construction of $r p o Q$ overexpression strains}

A full length (882 base pairs) copy of the A. salmonicida $r p o Q$ gene was amplified by PCR using the primer pair pTM214-rpoQ fwd and pTM214-rpoQ rev, containing the SacI and SalI restriction sites, respectively (Table 2). The resulting PCR product and the pTM214 expression vector (provided by Dr. Tim Miyashiro) were digested using SacI and SalI restriction enzymes. The digested PCR product was cloned downstream of the tryptophan promoter in the pTM214 expression vector, replacing the native $m$ Cherry gene. The construct was transformed

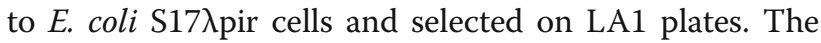
resulting plasmid is referred to as pTM214-rpoQ.

The pTM214-rpoQ and pTM214 (control vector) was transferred to LFI1238 and $\Delta$ litR by tri-parental mating using the conjugative helper strain CC118 $\lambda$ pir carrying pEVS104 (helper plasmid) as described by others [32], with some modifications. Briefly, E. coli S17入pir harboring pTM214-rpoQ or PIR2 harboring pTM214 and helper strain CC118 $\lambda$ pir carrying pEVS104 were grown to the mid-exponential phase at $37^{\circ} \mathrm{C}$. The recipient cells LFI1238 and $\Delta l i t R$ were grown to the early stationary phase. The donor, helper and recipient cells were mated at a 1:1:1 ratio after being harvested by centrifugation for $1 \mathrm{~min}$ at $4^{\circ} \mathrm{C}$ and washed with LB1 twice. The pelleted cells were mixed and spotted onto BA2.5 and incubated $\mathrm{ON}$ (overnight) at $16^{\circ} \mathrm{C}$. The spotted cells were resuspended in $2 \mathrm{ml} \mathrm{LB} 2.5$ and incubated $\mathrm{ON}$ at $12^{\circ} \mathrm{C}$ and $220 \mathrm{rpm}$. Transconjugants were selected on plates with chloramphenicol. The resulting strains are named LFI1238-pTM214, LFI1238-Ptrc-rpoQ, AlitRpTM214 and $\Delta$ litR-Ptrc-rpoQ.

\section{Construction of green fluorescent $A$. salmonicida LFI1238, $\triangle$ litR and $\triangle r p o Q$}

The pVSV102 plasmid encoding the green fluorescent protein (GFP) and kanamycin resistance was transferred from E. coli $\mathrm{DH} 5 \alpha \lambda$ pir to A. salmonicida LFI1238, $\Delta$ litR and $\triangle r p o Q$ using the conjugative helper strain CC118 $\lambda$ pir carrying pEVS104 as described above. The potential tagged strains were selected on BA2.5 after 5 days. The resulting strains were named LFI1238-pVSV102, AlitR-pVSV102 and $\triangle r p o Q-p V S V 102$. The GFP expression was confirmed microscopically using Nikon Eclipse TS100.

\section{Growth rate assay}

The overnight secondary cultures were diluted to $\mathrm{OD}_{600}$ of 0.05 in a total volume of $60 \mathrm{ml} \mathrm{SWT}$. The cultures were grown further in $250 \mathrm{ml}$ baffled flask at $8^{\circ} \mathrm{C}$ and $220 \mathrm{rpm}$. The optical density was measured every $3 \mathrm{~h}$ using Ultrospec 10 cell density meter (Amersham Biosciences).

\section{Motility assay}

The motility assay was performed using soft agar plates containing $0.25 \%$ agar and $2.5 \% \mathrm{NaCl}$ and with or without $1 \mathrm{mM}$ isopropyl $\beta$-D-1-thiogalactopyranoside (IPTG). The primary cultures were diluted 1:40 and incubated overnight at $12^{\circ} \mathrm{C}$ with agitation. The cultures were diluted to an $\mathrm{OD}_{600}$ of 0.4. Then $3 \mu \mathrm{l}$ of each culture was spotted on the soft agar plates and incubated at $4,8,12,14$ and $16^{\circ} \mathrm{C}$ for 5 days. The motility zones were monitored every $24 \mathrm{~h}$ for 5 days by measuring the diameter of the motile cells in the soft agar.

\section{Colony morphology and adhesion}

The colony morphology assay was carried out as described previously $[31,36]$. A $250 \mu \mathrm{l}$ of each bacterial culture was harvested by centrifugation, and the pellet was re-suspended in $250 \mu \mathrm{l} \mathrm{SWT}$. Then, $2 \mu \mathrm{l}$ of each culture was spotted onto SWT agar plates, and incubated at $4,8,12$, and $14^{\circ} \mathrm{C}$ for up to 3 weeks. The colonies were viewed microscopically with Zeiss Primo Vert and photographed with AxioCam ERc5s at $\times 4$ magnification. The same (three weeks old) colonies were also tested for their ability to adhere to the SWT agar. This was done by touching the colonies using a sterile plastic loop mainly as previously described [22], but the grading of the adherence was only recorded as "none" for smooth and creamy colonies, "weak" for slightly adherent and "strong" for colonies that were impossible to separate from the agar plate.

\section{Static biofilm assay}

The biofilm assay was performed as previously described [31]. The overnight secondary cultures were diluted to an $\mathrm{OD}_{600}$ of 1.3 in LB2.5 The cultures were further diluted 1:10 in SWT and a total volume of $300 \mu \mathrm{l}$ was added to each well in flat-bottom, non-tissue culturetreated Falcon 24-well plates (BD Bioscience). For the overexpression biofilm assay a total of $1 \mathrm{mM}$ IPTG was added. The plates were incubated statically at $4,8,12,14$ and $16^{\circ} \mathrm{C}$, for $72 \mathrm{~h}$ and the biofilm was visualized using Nikon Eclipse TS100 microscope at 10× magnification and photographed with Nikon DS-5Mc.

\section{Phylogenic analyses and software}

The amino acid sequences were aligned using ClustalW. The aligned sequences were then used to construct a neighbor-joining (NJ) tree using the MEGA version 7.0 [37]. Gaps in pairwise sequence comparison were deleted and the p-distance model was used. Bootstrap analyses with 500 replicates were conducted to provide confidence levels for the tree topology. Search for 
conserved sigma factor domains was performed using Pfam at EMBL-EBI (https://pfam.xfam.org/).

\section{Results}

Our previous studies show that $A$. salmonicida LitR is involved in regulating a number of activities that may be important for host interactions [22], and by using microarray we identified a number of genes regulated by LitR [31]. The regulation of LitR on downstream genes could proceed either directly or indirectly. One of the genes found to be regulated by LitR was the rpoQ gene (VSAL_IIO319). We therefore sought to analyze the role of RpoQ in the different phenotypes known to be regulated by LitR and QS in A. salmonicida. To this end we constructed an in-frame deletion mutant $(\triangle r p o Q)$ of the wild-type strain LFI1238 by removing 254 of the 294 amino acids in RpoQ. A complementation mutant $\left(\Delta r p o Q_{c}\right)$ was constructed to verify whether the observed phenotypes were due to the mutation of rpoQ. We do not expect the in-frame deletion $(\triangle r p o Q)$ or the insertion $(\Delta r p o Q c)$ of $r p o Q$ to have any polar effect(s) on downstream genes. However, it should be noted that this possibility cannot be excluded since the expression of the downstream genes in the operon was not analyzed in this work. Since temperature is an important factor involved in regulating AHL production and phenotypes related to QS in A. salmonicida $[22,31]$, the experiments were performed at different temperatures $\left(4-16^{\circ} \mathrm{C}\right)$.

\section{Deletion of rpoQ does not alter the growth of $A$. salmonicida}

To analyze if the rpoQ mutation affected the vitality of A. salmonicida LFI1238, a growth curve assay was performed. The bacterial growth of all strains (LFI1238, $\triangle r p o Q$ and the complementary strain) was monitored in triplicate at $8^{\circ} \mathrm{C}$ for $72 \mathrm{~h}$. The $\triangle r p o Q$ mutant showed the same growth rate as the wild-type strain LFI1238 and the complementary strain $\Delta r p o Q_{c}$ (Additional file 1: Figure S1).

\section{RpoQ shows temperature dependent rugose colony morphology}

The ability to form rugose colonies and biofilm are often correlated features in vibrios [38-40], and a rugose colony phenotype usually indicates high production of exopolysaccharides [39].

To compare colony morphologies of the wild-type LFI1238, and the $\triangle r p o Q$ and $\Delta l i t R$ mutants a spot colony assay was performed on SWT agar incubated at different temperatures $\left(4\right.$ to $14^{\circ} \mathrm{C}$ ). The LFI1238 produced smooth colony morphology at all temperatures as previously reported [31]. The $\triangle r p o Q$ mutant started to form wrinkled colonies after 7 days of incubation, and at day 12 a strong rugose colony morphology with wrinkled edges was observed after growth at 4 and $8^{\circ} \mathrm{C}$ (Fig. 1). When incubated at $12^{\circ} \mathrm{C}$, the $\triangle r p o Q$ colony remained smooth in the central part whereas the edges became wrinkled. No wrinkling was observed for $\triangle$ rpoQ at $14^{\circ} \mathrm{C}$. The $\Delta$ litR mutant was used as positive control [31] and, compared to $\triangle r p o Q$, it showed a weaker rugose colony morphology. A strong $\triangle$ litR rugose colony morphology similar to the wrinkled $\triangle r p o Q$ colonies was observed after 3 weeks (Additional file 2: Figure S2). As previously reported the wrinkling of $\Delta l i t R$ colonies is absent after growth at $14^{\circ} \mathrm{C}$ [31].

The wrinkled colonies formed by the $\triangle r p o Q$ and $\Delta l i t R$ mutants were found to be adhesive on the SWT agar, and the adhesiveness was stronger at low temperatures $\left(4\right.$ to $\left.8^{\circ} \mathrm{C}\right)$. No colonies were adhesive after growth at $14^{\circ} \mathrm{C}$ (Additional file 3: Table S1). The complementary strain $(\triangle r p o Q c)$ behaved similar to the wild-type and produced non-adhesive, smooth and creamy colonies at all temperatures.

\section{RpoQ is involved in biofilm formation}

In order to investigate whether rpoQ is involved in biofilm formation, the $\triangle r p o Q$ mutant was allowed to form biofilm in SWT medium at different temperatures using static conditions (Additional file 4: Figure S3). To better visualize the biofilm, GFP-tagged strains were used. The tagged strains were constructed by transferring a constitutive GFP expressing plasmid (pVSV102) into the different mutants and the wild-type strain. As shown in Fig. 2, $\triangle r p o Q$ produced a biofilm at 8 and $14^{\circ} \mathrm{C}$, which could be clearly visualized after $72 \mathrm{~h}$. Little or no biofilm was observed at $16^{\circ} \mathrm{C}$ for the different strains. The biofilm produced by the $\triangle r p o Q$ mutant does not show large mushroom shaped structures similar to those produced by $\Delta l i t R$ (Fig. 2 and [31]); instead the $\Delta r p o Q$ mutant formed a more regular and flat biofilm with smaller micro-colonies and structures. Above the microscopically visual $\triangle r p o Q$ biofilm structures is a thick and slimy extracellular matrix without or with few embedded bacteria (Additional file 5: Figure S4). The complementary strain $\triangle r p o Q c$ behaved similar to the wild-type, whereas the double mutant $\Delta$ litR-rpo $Q^{-}$produced a biofilm with mushroom structure similar to the one produced by the $\triangle l i t R$ mutant (Additional file 4: Figure S3).

\section{RpoQ regulates motility in $A$. salmonicida}

The flagellum is required for motility of bacteria, mediating their movements towards favorable environments or away from harmful conditions [41, 42]. Previous studies have shown that $A$. salmonicida is more motile at $12^{\circ} \mathrm{C}$ than at $4^{\circ} \mathrm{C}$, and that LitR is a negative regulator of motility [22]. Here we analyzed the influence of RpoQ on the motility of A. salmonicida at different temperatures $\left(4\right.$ to $\left.16^{\circ} \mathrm{C}\right)$. Deletion of rpoQ resulted in a strain 

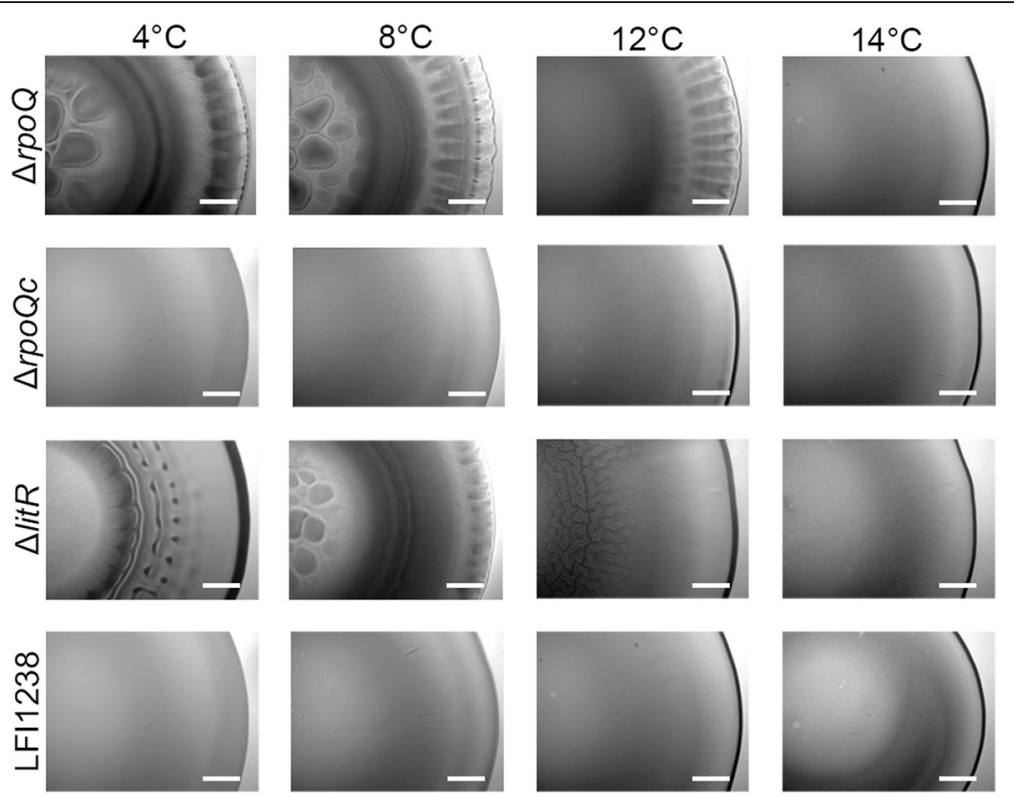

Fig. 1 Colony morphology of $\triangle r p o Q, \Delta r p o Q_{c} \Delta /$ itR and LFl1238 at different temperatures. The colonies were allowed to form on SWT plates for 12 days at $4,8,12$ and $14^{\circ} \mathrm{C}$. The colonies were viewed in a Zeiss Primo Vert microscope at $4 \times$ magnification. Scale bars represent $0.5 \mathrm{~mm}$

with reduced motility compared to the wild-type and the $\Delta$ litR mutant at all tested temperatures (Fig. 3 and Additional file 6: Table S2). After 5 days of incubation at $4^{\circ} \mathrm{C}$ the $\triangle r p o Q$ mutant was almost non-motile and the motility zone was only $6.0 \pm 1.0 \mathrm{~mm}$. At higher temperatures $\left(8\right.$ to $\left.16^{\circ} \mathrm{C}\right)$ the motility of the $\triangle r p o Q$ mutant was between 36 and $51 \%$ compared to the motility of wild-type. Hence, the incubation temperature did not seem to affect the regulatory effect of RpoQ on the motility. Similar to the wild-type and $\Delta l i t R$, the $\Delta r p o Q$ mutant shows highest motility at $14^{\circ} \mathrm{C}$. The $\triangle r p o Q c$ behaved similar to the wild-type (Fig. 3a and b).
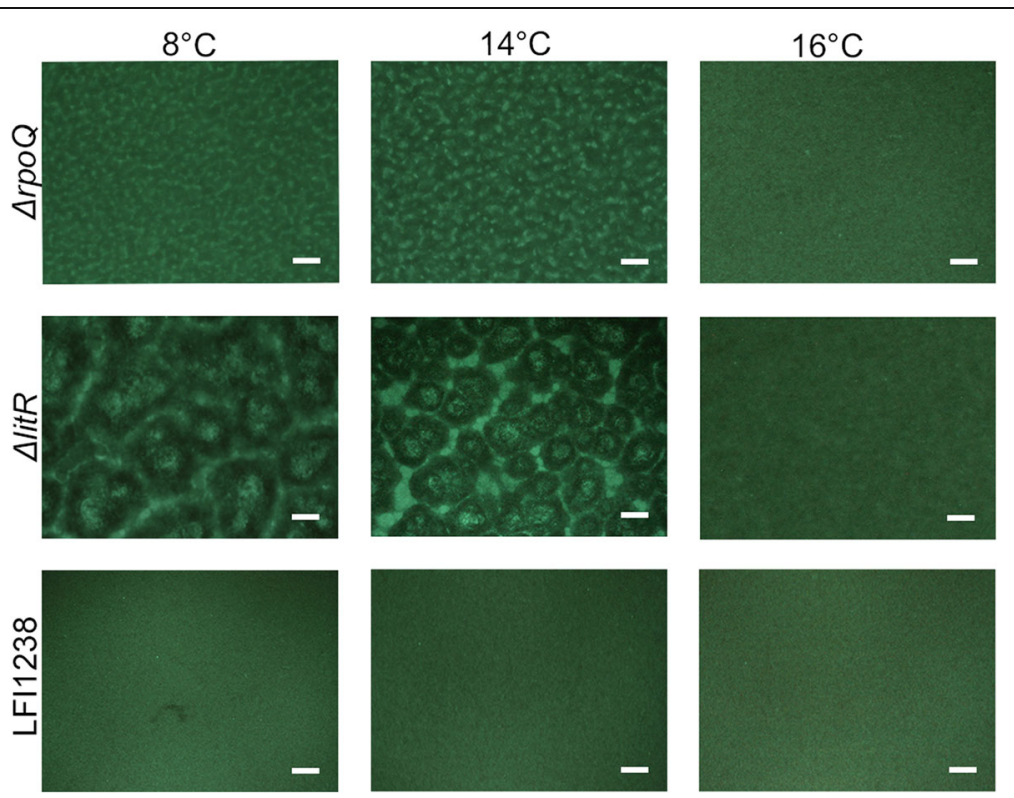

Fig. 2 Biofilm formation of GFP-tagged $\Delta r p o Q, \Delta l i t R$ and LFI1238 at different temperatures. The GFP tagged strains (LFI1238-pVSV102, ArpoQ-pVSV102 and $\Delta /$ itR-pVSV102) were allowed to form biofilms in SWT media at 8,14 and $16^{\circ} \mathrm{C}$. The biofilms were viewed, after $72 \mathrm{~h}$ of incubation, in a Nikon Eclipse TS100 microscope at 10x magnification and photographed with Nikon DS-5Mc. Scale bars represent $20 \mu \mathrm{m}$ 


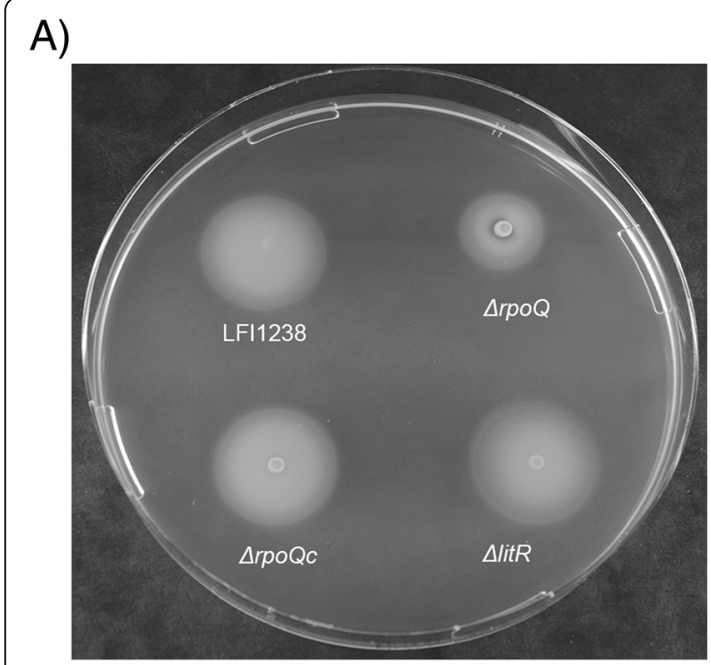

\section{B)}
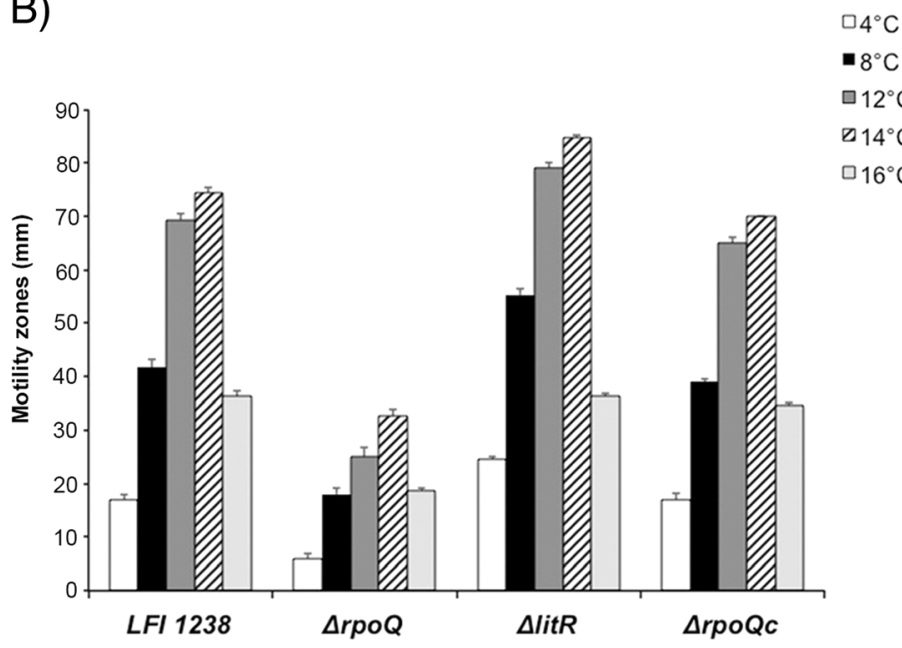

Fig. 3 Motility of $L F \mid 1238, \Delta r p o Q, \Delta r p o Q_{c}$ and $\Delta /$ litR at different temperatures. a Soft agar plate showing motility zones of $L F \mid 1238, \Delta r p o Q, \Delta r p o Q_{c}$ and $\Delta$ litR after 5 days incubation at $8^{\circ} \mathrm{C}$. $\mathbf{b}$ Motility zones $(\mathrm{mm})$ of $L F \mid 1238, \Delta r p o Q, \Delta l i t R$, and $\Delta r p o Q_{c}$ measured after 5 days incubation at different temperatures $\left(4-16^{\circ} \mathrm{C}\right)$. The error bars present the standard deviation of biological triplicate

\section{RpoQ is a negative regulator of biofilm}

RpoQ is believed to function downstream of LitR in the QS cascade in A. salmonicida [31], and as shown above, deletion of $r p o Q$ resulted in increased biofilm formation (Fig. 2). Hence, it was of interest to examine the influence of overexpressing $r p o Q$ on the $\triangle$ litR biofilm formation. For this purpose, the control vector (pTM214) and the inducible rpoQ vector (pTM214-rpoQ) were separately transferred to the $\Delta l i t R$ mutant strain and the wild-type LFI1238 by conjugation. The biofilm assay was performed as before in SWT medium $\left(4\right.$ to $16^{\circ} \mathrm{C}$ ) but with $1 \mathrm{mM}$ IPTG to induce expression of $r p o Q$.

As shown in Fig. 4, overexpression of rpoQ disrupted or inhibited the biofilm formation produced by $\Delta l i t R$ ( $\triangle$ litR-Ptrc-rpoQ at 4 to $14^{\circ} \mathrm{C}$ ) leaving small aggregates in the wells, whereas the $\Delta l i t R$ biofilm formation was unaffected by the presence of the control vector (AlitR-pTM214) at all temperatures. Biofilm formation does not occur at $16^{\circ} \mathrm{C}$, and hence no effects of the overproduced rpoQ was observed. Neither was any
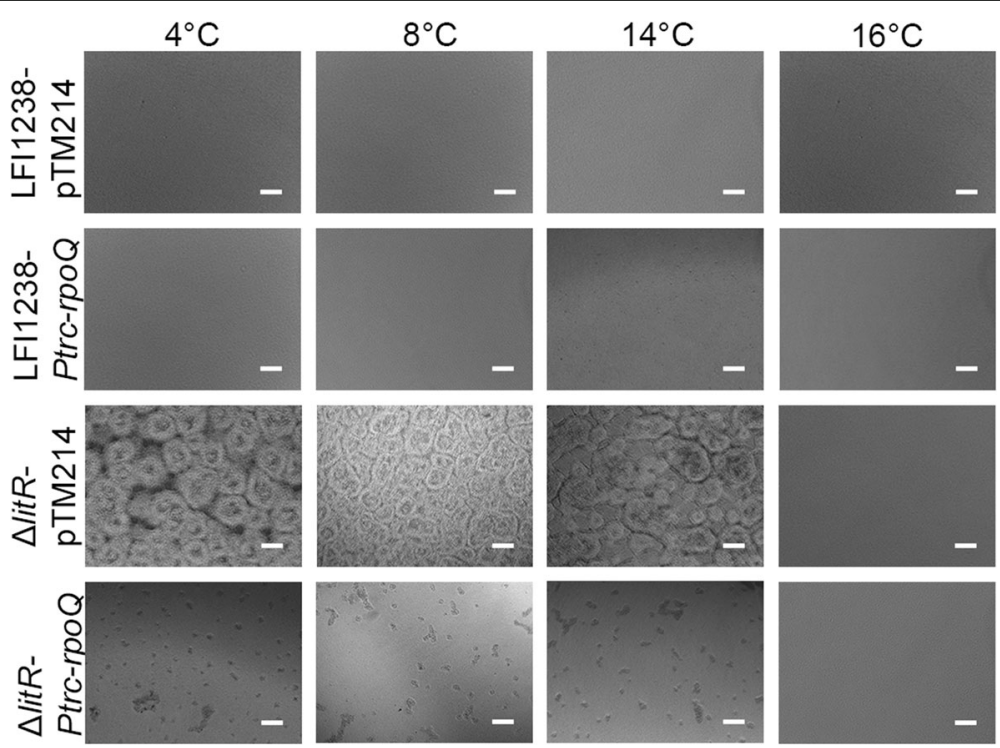

Fig. 4 The effect of the RpoQ on the biofilm formation of LFI1238 and $\Delta$ litR. The biofilms of LFI1238 and $\Delta$ litR harboring the pTM214 (control vector) and LFI1238 and $\Delta$ litR harboring the $P_{\text {trc }}$ rpoQ (rpoQ overexpression vector) were allowed to form in SWT medium supplemented with $1 \mathrm{mM}$ IPTG. The biofilms were incubated for $72 \mathrm{~h}$ at different temperatures $\left(4\right.$ to $16^{\circ} \mathrm{C}$ ). The biofilms were viewed in Nikon Eclipse TS100 microscope at 10x magnification and photographed with Nikon DS-5Mc. Scale bars represent $20 \mu \mathrm{m}$ 
changes observed when rpoQ was overexpressed in wild-type cells (LFI1238-Ptrc-rpoQ) (Fig. 4).

\section{Overexpression of RpoQ decreases motility in $A$. salmonicida}

In the experiments performed above we show that rpoQ is required for full wild- type motility at all temperatures (Fig. 3) and that overexpression of $r p o Q$ has a negative effect on the biofilm forming ability of the $\Delta$ litR mutant (Fig. 4). It therefore was of interest to analyze if overexpressed $r p o Q$ also affected the motility of the wild-type and the $\Delta l i t R$ mutant. As shown in Fig. 5, overexpression of rpoQ repressed the motility in both strains. Most notable, overexpression of $r p o Q$ in the wild-type resulted in a completely non-motile strain when incubated at 4 and $8^{\circ} \mathrm{C}$, and the size of the spotted LFI1238-Ptrc-rpoQ colony $(5 \mathrm{~mm})$ did not change at any of the two temperatures during the 5 days of the experiment (Fig. $5 \mathrm{a}$ and b). At 12,14 and $16^{\circ} \mathrm{C}$ small motility zones (7$9 \mathrm{~mm}$ ) were observed for LFI1238-Ptrc-rpoQ showing that overexpression of $r p o Q$ in the wild-type does not result in complete shutdown of the motility at these temperatures. Overexpression of $r p o Q$ in the $\triangle$ litR also resulted in clearly diminished motility zones at all temperatures (Fig. 5b and Additional file 7: Table S3).

\section{Discussion}

Bacteria continually face changes in their environment such as temperature fluctuations, nutrient accessibility and $\mathrm{pH}$ changes. In order to adapt to these changes and often challenging conditions, bacteria have developed various responses. Alternative sigma factors such as RpoS provide a main line of responses to changes in the environment by altering gene transcription [43, 44]. Several studies have shown a connection between RpoS and QS in different vibrios [38, 45-48]. When Cao et al. (2012) described the alternative sigma factor RpoQ in A. fischeri a homologue was only found in A. salmonicida [28]. However, since then the genomes of $A$. wodanis [49] and $A$. logei (A.logei S5-186 GeneBank accession no. AJY02000108.1) have become available. Analysis show that they also encode an RpoQ homolog with four conserved domains $\left(\sigma^{70}\right.$ regions 1-4). RpoQ of A. salmonicida shares a high amino acid sequence identity (99\%) with its homolog in $A$. logei whereas the amino acid sequence identity is $72 \%$ with A. fischeri and $69 \%$ with A. wodanis. Region 2 and region 4 of the putative RpoQ are well conserved between the four species, whereas region 3 is less conserved (Additional file 8: Figure S5).

RpoQ is regulated by LuxO through LitR in A. fischeri [28]. Similarly, our previous microarray results suggested that LitR is a positive regulator of RpoQ in A. salmonicida [31]. In the study presented here, we show that RpoQ is involved in regulation of colony morphology, adhesion, biofilm and motility similar to LitR. However, since RpoQ is suspected to act downstream of the master regulator LitR, one can expect that the $\Delta$ litR mutant expresses phenotypes that are independent of RpoQ regulation.

The $\triangle r p o Q$ mutant demonstrated a stronger and an earlier onset of the rugose colony morphology as compared to the $\Delta l i t R$ mutant. A rugose colony phenotype usually develops when the bacteria produce high amounts of polysaccharides, suggesting that more polysaccharides
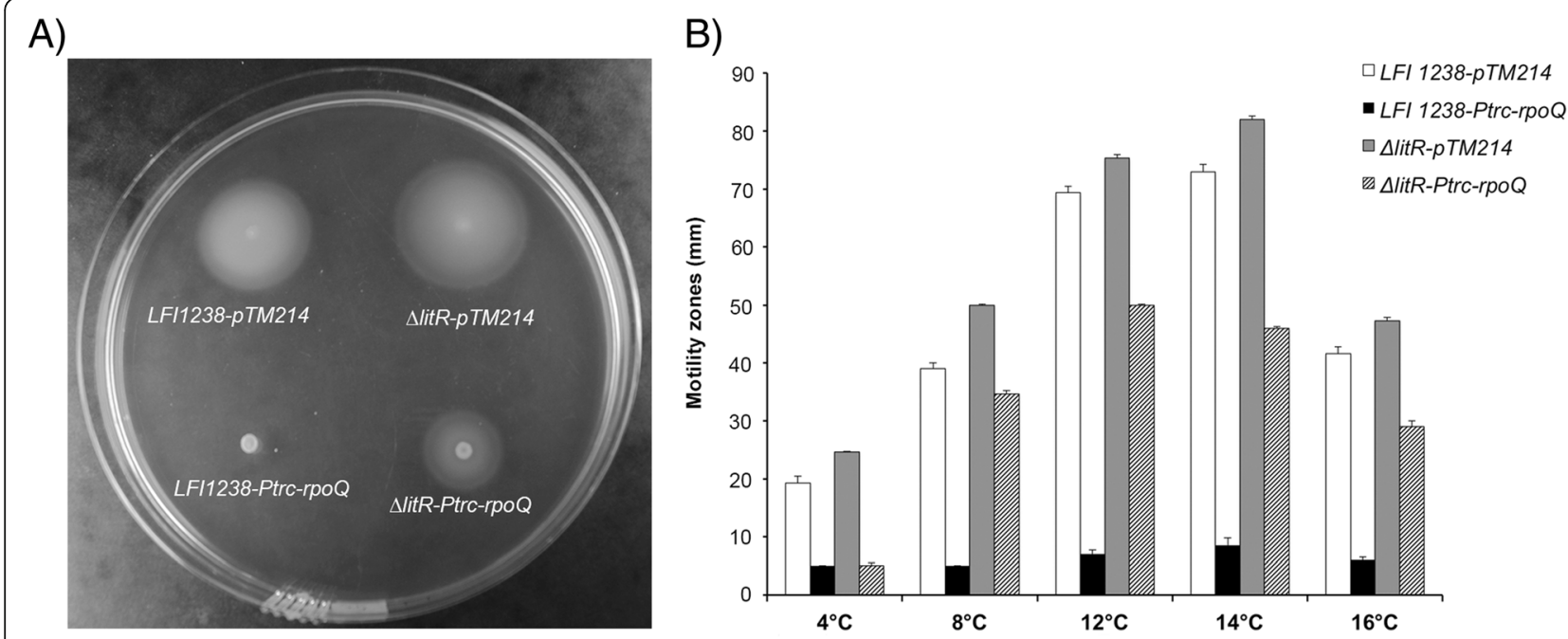

Fig. 5 Motility assay on soft agar supplemented with $1 \mathrm{mM} \mathrm{IPTG}$. a Soft agar plate showing motility zones of LFI1238 and $\Delta$ litR harboring the pTM214 (control vector) and LFI1238 and $\Delta$ litR harboring the $P_{\text {trc }}$-rpoQ (rpoQ overexpression vector) at $8^{\circ} \mathrm{C}$ after 5 days. b Motility zones (mm) of LFI1238-pTM214, $\Delta$ litR-pTM214, LFI1238- $P_{\text {trc }}-r p o Q$ and $\Delta$ litR- $P_{\text {trc }}-r p o Q$ after 5 days of incubation at temperatures ranging from 4 to $16^{\circ} \mathrm{C}$. The error bars present the standard deviation of biological triplicate 
are made by the $\triangle r p o Q$ mutant. We know from our previous work that LitR represses the expression of the symbiosis polysaccharide (syp) operon, and that inactivation of syp (sypC, sypP and sypQ) in the $\Delta$ litR mutant results in smooth colonies [31]. Hence, it is likely that LitR performs its activity on syp through RpoQ and that activation of RpoQ leads to a strong(er) repression of syp. The weaker rugose colony morphology of the $\Delta l i t R$ mutant may be due to low levels of LitR-independent rpoQ expression, consistent with our previous microarray results that show expression of rpoQ in the $\Delta l i t R$ mutant [31]. Hence, some repression of syp via RpoQ probably occurs in the $\Delta l i t R$ mutant. Whereas in the $\triangle r p o Q$ mutant there is zero expression of rpoQ resulting in no or low syp repression and stronger rugosity.

Both LitR and RpoQ are negative regulators of biofilm formation in $A$. salmonicida. However, the $\triangle r p o Q$ mutant formed a biofilm morphologically different from the $\Delta l i t R$ mutant. The biofilm produced by the $\Delta r p o Q$ was less mature and relatively flat and compact, without the large mushroom structures exhibited by the $\Delta l i t R$ mutant. Additionally, the biofilm produced by $\triangle r p o Q$ contained a heavy and slimy extracellular matrix substance above the biofilm cells attached to the substratum (Additional file 5: Figure S4). This slimy matrix is likely due to high amounts of polysaccharides (i.g. syp expression as discussed above) that are common components of the extracellular matrix of biofilms, together with proteins and eDNA [50]. When we previously analyzed the $\triangle l i t R$ biofilm we found that major components were polysaccharides and proteins, and by using electron microscopy we were able to see a network of fibers that connected biofilm cells together. The microarray analysis identified, in addition to syp, some lipoprotein, pili, flagella, and curli genes that were upregulated in the $\triangle$ litR mutant [31]. Hence, LitR may repress some lipoproteins or filament structures needed to build up this mushroom-shaped biofilm architecture. Thus, one explanation for the observed biofilm morphology of the $\triangle r p o Q$ mutant may be that polysaccharide production is obtained through expression of syp, whereas expression of a functional LitR down-regulates genes involved in building mushroom shaped structures. When we inactivated syp in the $\Delta$ litR mutant we found that although the rugose colony morphology reverted to wild-type morphology (smooth), some biofilm formation still occurred when using SWT medium [31]. Indeed, the biofilms produced by the $\Delta l i t R s y p^{-}$mutants resembles the biofilm produced by $\triangle r p o Q$ but without the slimy extracellular matrix. We therefore believe that the pathway through which LitR represses genes responsible for building the mushroom-shaped structures is different from the pathway through which LitR represses syp (via RpoQ) resulting in rugose colony morphology (Fig. 6).
Both mutants produce biofilms that are loosely attached; however, in contrast to the $\Delta l i t R$ biofilm, the $\triangle r p o Q$ biofilm is not able to withstand the washing steps required after staining with crystal violet. To our knowledge RpoQ, has not been shown to be involved in biofilm formation of $A$. fischeri or any other aliivibrios. However, studies have shown that RpoS is able to enhance or repress biofilm formation in E. coli and other bacteria [51-53]. Additionally, RpoS has been shown to be involved in cell attachment and the maturation of biofilm $[30,54,55]$, and inactivation RpoX in V. alginolyticus results in cells with decreased ability to form biofilm [29]. Likewise, inactivation of $r p o Q$ in A. salmonicida may have reduced the ability of the bacteria to attach to the abiotic surface and to build a mature biofilm. Another explanation is that the $\triangle r p o Q$ biofilm contains a higher amount of a heavy, extracellular, slimy polysaccharide matrix that tears the biofilm away from the substratum when the medium or wash solutions is being poured out or a combination of both.

Thus, as shown in Fig. 6 we propose that RpoQ and LitR function in the same pathway, where RpoQ functions

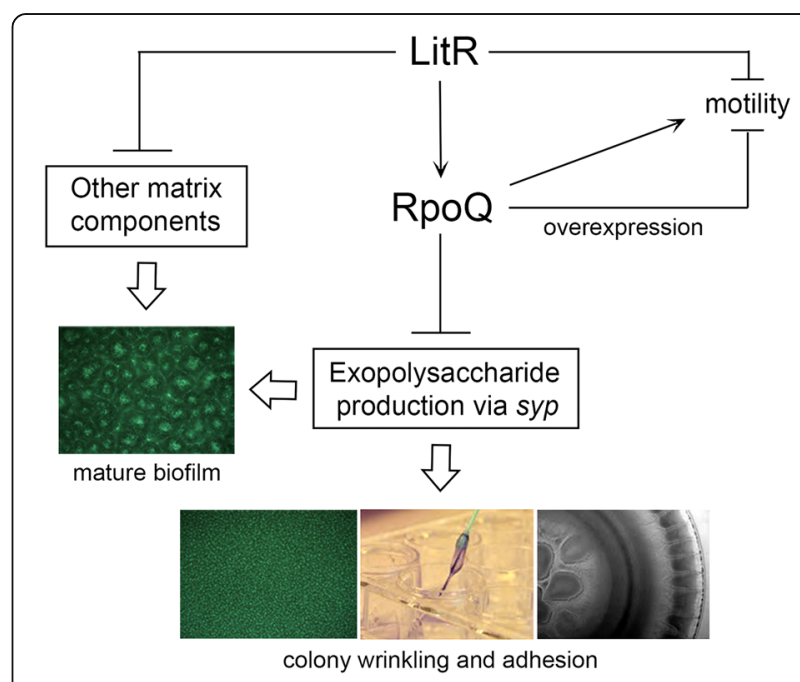

Fig. 6 Proposed model for regulation of QS related phenotypes in A. salmonicida. At high cell densities, LitR is produced in response to AHLs and acts as a positive regulator of rpoQ expression. LitR, probably via RpoQ, downregulates motility and expression of exopolysaccharides. The $\Delta$ lit $R$ mutant shows a mature biofilm with mushroom shaped structures, whereas the $\triangle r p o Q$ biofilm is more flat and regular. Thus, in addition to repression of exopolysaccharides via RpoQ, LitR represses other biofilm matrix components independent of RpoQ that are required for building mature mushroom structures (e.g. lipoproteins, protein filaments). Therefore, at high cell densities both RpoQ dependent and independent processes are needed for down regulation of the mature biofilm. The $\triangle r p o Q$ mutant shows decreased motility suggesting that RpoQ may also act as a positive activator of motility. Arrows and lines with bar ends indicate pathways of positive and negative regulation, respectively, and may consist of several steps. The thicker, empty arrows indicate the resulting phenotypes 
downstream of the LitR and is involved in repression of biofilm and the wrinkled colony morphology in A. salmonicida. The negative regulation cascade of extracellular polysaccharide matrix from LitR to the syp operon is probably operated through RpoQ, either directly or indirectly. The phenotypes are likely regulated in a cell density manner as previously discussed, where the development of a mushroom shaped biofilm structures and wrinkled colony morphology are initiated when neither AinS or LuxI AHLs are present at low cell density [19, 22, 31]. At high cell density when AHLs are produced, LitR represses genes required for building a mature biofilm structure, and activates $r p o Q$ leading to repression of syp.

Inactivation of either rpoQ or litR had the opposite effect on motility in A. salmonicida. Unlike the $\Delta l i t R$ mutant, which is more motile than the wild-type strain, the $\triangle r p o Q$ mutant exhibited significantly reduced motility. The complementary strain $\Delta r p o Q c$ showed wild-type motility, suggesting that the termination of motility is due to rpoQ deletion and not to other factors. Reduced motility due to disruption of sigma factors has been reported for other bacteria, and inactivation of rpoS in $Y$. pseudotuberculosis results in decreased motility due to downregulation of the flagella master regulatory gene flhDC [51]. Thus, RpoQ may work in a similar manner by altering transcription of genes responsible for flagellar assembly or flagellar biosynthesis in A. salmonicida. Flagellum-mediated motility is important for specific stages of biofilm formation and surface attachment in several bacteria [56-58], and disruption of flagella biosynthesis is known to decrease attachment and alter biofilm architecture [59-62]. For example, loss of motility in E. coli affected the biofilm architecture, where poorly motile strains formed flatter biofilms compared to highly motile strains, which displayed more mature vertical biofilm structures [63]. Thus, it is tempting to speculate that the decreased motility of the $\triangle r p o Q \mathrm{mu}-$ tant resulted in cells with reduced ability to attach and form mature biofilms.

Furthermore, overexpression of rpoQ resulted in non-motile wild-type cells and $\Delta l i t R$ cells with reduced motility. These results are similar to those obtained with A. fischeri, where the overexpression of rpoQ in the wild-type and $\Delta l i t R$ mutant resulted in non-motile strains [28]. The finding that both deletion and overexpression of rpoQ in A. salmonicida resulted in bacteria with reduced motility is interesting, but at the same time difficult to interpret. We know that RpoQ functions downstream of LitR and that LitR is a negative regulator of motility at high cell density [22, 31]. Thus, we may have expected to observe a similar effect on motility when we knocked out rpoQ. However, the $\triangle r p o Q$ mutant show decreased motility compared to the wild-type indicating that RpoQ is a positive regulator of motility
(Fig. 6). This may suggest that at low cell densities some litR independent expression of rpoQ occurs and that RpoQ activates genes involved in flagellar biosynthesis. As the cell population increases litR will be expressed leading to increased levels of RpoQ. High RpoQ levels (overexpression of $r p o Q$ ) then turns down motility probably by acting as an activator of genes involved in down regulation of the flagellar apparatus. Hence, RpoQ probably controls genes responsible for both promoting or repressing motility depending on growth phase, environmental conditions and stress factors. Our results show that regulation of motility in A. salmonicida is complex similar to other vibrios [64] and probably involves several regulatory genes and factors, which is still unrevealed.

Temperature is an important factor in developing cold-water vibriosis and for production of AHLs in $A$. salmonicida. When the bacteria is grown at temperature above the disease limit $\left(16^{\circ} \mathrm{C}\right)$, the production of AHLs is nearly absent [19]. Our results from the biofilm and colony morphology assays show that the $\triangle r p o Q$ mutant behaves as the wild-type strain and the $\Delta l i t R$ mutant when the assays are performed at $16^{\circ} \mathrm{C}$, and neither of the strains forms rugose colonies or biofilm at this temperature. This shows that RpoQ, similar to LitR, represses formation of biofilm and rugose colonies more at low temperatures $\left(4-14^{\circ} \mathrm{C}\right)$, and at $16^{\circ} \mathrm{C}$ the effect of the $r p o Q$ deletion is absent with regard to these phenotypes. Interestingly, this temperature effect was not observed when the motility of the $\triangle r p o Q$ mutant was analyzed, and at $16^{\circ} \mathrm{C}$ the motility of the $\triangle r p o Q$ mutant was still clearly reduced compared to the wild-type. This implies that RpoQ is expressed and is able to regulate motility in A. salmonicida at temperatures above the limit for developing cold water vibriosis, and at conditions when AHL concentrations are expected to be low.

\section{Conclusion}

In this work we have shown that the alternative sigma factor RpoQ regulates motility, colony morphology and biofilm formation in A. salmonicida. This broad range of different phenotypes suggests that RpoQ is involved in a regulatory hierarchy influencing expression of a large panel of genes. Overexpression of RpoQ led to disruption of the biofilm produced by $\Delta l i t R$, paralyzed the motility of the wild-type A. salmonicida and caused a reduction in $\Delta l i t R$ motility. These findings confirm that the RpoQ is a novel factor in the QS and functions downstream of the LitR. However, further studies are needed to understand exactly how LitR and RpoQ work together or independently to regulate the QS dependent phenotypes investigated here, and to identify genes regulated by RpoQ. 


\section{Additional files}

Additional file 1: Figure S1. The figure shows growth curves of $A$. salmonicida wild type and rpoQ mutants. (DOCX $126 \mathrm{~kb}$ )

Additional file 2: Figure S2. The figure shows colony morphology of $\Delta$ litR after 3 weeks of incubation. (DOCX $101 \mathrm{~kb}$ )

Additional file 3: Table S1. The table lists grading of adherence of $A$ salmonicida wild-type and mutants on SWT agar. (DOCX 16 kb)

Additional file 4: Figure S3. The figure shows biofilm formation of $A$. salmonicida wild-type LFI1238 and mutants. (DOCX 642 kb)

Additional file 5: Figure S4. The figure shows the slimy extracellular matrix formed by $\triangle r p o Q$ in the biofilm assay. (DOCX $701 \mathrm{~kb}$ )

Additional file 6: Table S2. The table lists motility zones of LFI1238, $\triangle r p o Q, \Delta r p o Q c$ and $\Delta / i t R$ formed on soft agar plates. (DOCX $15 \mathrm{~kb}$ )

Additional file 7: Table S3. The table lists motility zones formed on soft agar plates supplemented with 1 mM IPTG. (DOCX 16 kb)

Additional file 8: Figure S5. The figure shows alignment and phylogeny of RpoQ, RpoS and RpoX. (DOCX 699 kb)

\section{Abbreviations}

IPTG: Isopropyl $\beta$-D-1-thiogalactopyranoside; min: Minutes; OD 600 : Optical density measured at $600 \mathrm{~nm}$; ON: Overnight; PCR: Polymerase chain reaction; QS: Quorum sensing; rpm: Rounds per minute

\section{Acknowledgements}

We thank Dr. Debra Milton (Umeå University) for the pDM4 and pNQ705 plasmids, Dr. Eric V. Stabb (University of Georgia) and Dr. Tim Miyashiro (Penn State University) for the pVSV102, pTM214 and pEVS104 plasmids. We also thank Prof. Richard Engh (UiT The Arctic University of Norway) for proofreading parts of this manuscript.

\section{Funding}

This work was financed by UiT The Arctic University of Norway. UiT was not involved in designing the study, analysis, collection, data interpretation and in writing the manuscript. The publication charges for this article have been funded by a grant from the publication fund of UiT The Arctic University of Norway.

\section{Availability of data and materials}

The datasets used and/or analyzed during the current study are available from the corresponding author on reasonable request.

\section{Authors' contributions}

MK, $\mathrm{HH}$ and NPW conceived and designed the experiments. MK and $\mathrm{HH}$ constructed the mutants. MK constructed the GFP-tagged and overexpression mutants. MK performed the motility, morphology and biofilm assays. $\mathrm{MK}$ and $\mathrm{HH}$ wrote the paper. All authors read and approved the final manuscript.

\section{Ethics approval and consent to participate}

The experimental work carried in this study does not have any human or animal subjects. We do not see any ethical issues.

\section{Consent for publication}

Not applicable.

\section{Competing interests}

The authors declare that they have no competing interest.

\section{Publisher's Note}

Springer Nature remains neutral with regard to jurisdictional claims in published maps and institutional affiliations.
Received: 16 April 2018 Accepted: 4 September 2018

Published online: 12 September 2018

\section{References}

1. Thompson FL, lida T, Swings J. Biodiversity of vibrios. Microbiol Mol Biol Rev. 2004;68(3):403-31.

2. Urbanczyk H, Ast JC, Higgins MJ, Carson J, Dunlap PV. Reclassification of Vibrio fischeri, Vibrio logei, Vibrio salmonicida and Vibrio wodanis as Aliivibrio fischeri gen. Nov., comb. nov., Aliivibrio logei comb. nov., Aliivibrio salmonicida comb. nov. and Aliivibrio wodanis comb. nov. Int J Syst Evol Microbiol. 2007:57(Pt 12):2823-9.

3. Egidius E, Andersen K, Clausen E, Raa J. Cold-water vibriosis or "Hitra disease" in Norwegian salmonid farming. J Fish Dis. 1981:4(4):353-4.

4. Holm K, Strøm E, Stensvaag K, Raa J, Jørgensen T. Characteristics of a Vibrio sp. associated with the "Hitra disease" of Atlantic Salmon in Norwegian fish farms. Fish Pathology. 1985;20(2-3):125-9.

5. Egidius E, Wiik R, Andersen K, Hoof KA, Hjeltnes B. Vibrio salmonicida sp. nov., a new fish pathogen. Int J Syst Evol Microbiol. 1986;36(4):518-20.

6. Miller MB, Bassler BL. Quorum sensing in bacteria. Annu Rev Microbiol. 2001:55:165-99.

7. $\mathrm{Ng} \mathrm{WL}$, Bassler BL. Bacterial quorum-sensing network architectures. Annu Rev Genet. 2009:43:197-222.

8. Ruby EG. Lessons from a cooperative, bacterial-animal association: the Vibrio fischeri-Euprymna scolopes light organ symbiosis. Annu Rev Microbiol. 1996; 50:591-624.

9. Hastings JW, Nealson KH. Bacterial bioluminescence. Annu Rev Microbiol. 1977;31:549-95

10. Bassler BL, Wright M, Showalter RE, Silverman MR. Intercellular signalling in Vibrio harveyi: sequence and function of genes regulating expression of luminescence. Mol Microbiol. 1993;9(4):773-86.

11. Lupp C, Ruby EG. Vibrio fischeri uses two quorum-sensing systems for the regulation of early and late colonization factors. J Bacteriol. 2005: 187(11):3620-9.

12. Fidopiastis PM, Miyamoto CM, Jobling MG, Meighen EA, Ruby EG. LitR, a new transcriptional activator in Vibrio fischeri, regulates luminescence and symbiotic light organ colonization. Mol Microbiol. 2002;45(1):131-43.

13. Miyashiro T, Wollenberg MS, Cao X, Oehlert D, Ruby EG. A single arr gene is necessary and sufficient for LuxO-mediated regulation in Vibrio fischeri. Mol Microbiol. 2010;77(6):1556-67.

14. Lupp C, Ruby EG. Vibrio fischeri LuxS and AinS: comparative study of two signal synthases. J Bacteriol. 2004;186(12):3873-81.

15. Miyashiro T, Ruby EG. Shedding light on bioluminescence regulation in Vibrio fischeri. Mol Microbiol. 2012:84(5):795-806.

16. Engebrecht J, Silverman M. Identification of genes and gene products necessary for bacterial bioluminescence. Proc Natl Acad Sci U S A. 1984; 81(13):4154-8.

17. Verma SC, Miyashiro T. Quorum sensing in the squid-Vibrio symbiosis. Int J Mol Sci. 2013:14(7):16386-401.

18. Hjerde E, Lorentzen MS, Holden MT, Seeger K, Paulsen S, Bason N, Churcher C, Harris D, Norbertczak H, Quail MA, et al. The genome sequence of the fish pathogen Aliivibrio salmonicida strain LFI1238 shows extensive evidence of gene decay. BMC Genomics. 2008:9:616.

19. Hansen H, Purohit AA, Leiros HK, Johansen JA, Kellermann SJ, Bjelland AM Willassen NP. The autoinducer synthases Luxl and AinS are responsible for temperature-dependent AHL production in the fish pathogen Aliivibrio salmonicida. BMC Microbiol. 2015;15:69.

20. Fidopiastis PM, Sørum H, Ruby EG. Cryptic luminescence in the cold-water fish pathogen Vibrio salmonicida. Arch Microbiol. 1999;171(3):205-9.

21. Hmelo LR. Quorum sensing in marine microbial environments. Annu Rev Mar Sci. 2017:9:257-81.

22. Bjelland AM, Sørum $H$, Tegegne DA, Winther-Larsen HC, Willassen NP Hansen $\mathrm{H}$. LitR of Vibrio salmonicida is a salinity-sensitive quorum-sensing regulator of phenotypes involved in host interactions and virulence. Infect Immun. 2012;80(5):1681-9.

23. Borukhov S, Nudler E. RNA polymerase holoenzyme: structure, function and biological implications. Curr Opin Microbiol. 2003;6(2):93-100.

24. Davis MC, Kesthely CA, Franklin EA, MacLellan SR. The essential activities of the bacterial sigma factor. Can J Microbiol. 2017;63(2):89-99.

25. Tripathi L, Zhang $Y$, Lin Z. Bacterial sigma factors as targets for engineered or synthetic transcriptional control. Front Bioeng Biotechnol. 2014;2:33. 
26. Boyd EF, Carpenter MR, Chowdhury N, Cohen AL, Haines-Menges BL, Kalburge SS, Kingston JJ, Lubin JB, Ongagna-Yhombi SY, Whitaker WB. Postgenomic analysis of members of the family Vibrionaceae. Microbiol Spectr. 2015:3:5.

27. Mandel MJ, Stabb EV, Ruby EG. Comparative genomics-based investigation of resequencing targets in Vibrio fischeri: focus on point miscalls and artefactual expansions. BMC Genomics. 2008;9:138.

28. Cao X, Studer SV, Wassarman K, Zhang Y, Ruby EG, Miyashiro T. The novel sigma factor-like regulator RpoQ controls luminescence, chitinase activity, and motility in Vibrio fischeri. mBio. 2012;3:e00285-11.

29. Paget MS, Helmann JD. The sigma70 family of sigma factors. Genome Biol. 2003;4(1):203.

30. Zhao JJ, Chen C, Zhang LP, Hu CQ. Cloning, identification, and characterization of the rpoS-like sigma factor rpoX from Vibrio alginolyticus. J Biomed Biotechnol. 2009;2009:126986.

31. Hansen H, Bjelland AM, Ronessen M, Robertsen E, Willassen NP. LitR is a repressor of syp genes and has a temperature-sensitive regulatory effect on biofilm formation and colony morphology in Vibrio (Aliivibrio) salmonicida. Appl Environ Microbiol. 2014;80(17):5530-41.

32. Stabb EV, Ruby EG. RP4-based plasmids for conjugation between Escherichia coli and members of the Vibrionaceae. Methods Enzymol. 2002;358:413-26.

33. Miyashiro T, Klein W, Oehlert D, Cao X, Schwartzman J, Ruby EG. The Nacetyl-D-glucosamine repressor NagC of Vibrio fischeri facilitates colonization of Euprymna scolopes. Mol Microbiol. 2011;82(4):894-903.

34. Shubeita HE, Sambrook JF, McCormick AM. Molecular cloning and analysis of functional CDNA and genomic clones encoding bovine cellular retinoic acid-binding protein. Proc Natl Acad Sci U S A. 1987;84(16):5645-9.

35. Milton DL, O'Toole R, Horstedt $P$, Wolf-Watz $H$. Flagellin a is essential for the virulence of Vibrio anguillarum. J Bacteriol. 1996;178(5):1310-9.

36. Lim B, Beyhan S, Meir J, Yildiz FH. Cyclic-diGMP signal transduction systems in Vibrio cholerae: modulation of rugosity and biofilm formation. Mol Microbiol. 2006:60(2):331-48.

37. Saitou N, Nei M. The neighbor-joining method: a new method for reconstructing phylogenetic trees. Mol Biol Evol. 1987;4(4):406-25.

38. Yildiz FH, Liu XS, Heydorn A, Schoolnik GK. Molecular analysis of rugosity in a Vibrio cholerae 01 El Tor phase variant. Mol Microbiol. 2004;53(2):497-515.

39. Yildiz FH, Schoolnik GK. Vibrio cholerae 01 El Tor: identification of a gene cluster required for the rugose colony type, exopolysaccharide production, chlorine resistance, and biofilm formation. Proc Natl Acad Sci U S A. 1999; 96(7):4028-33.

40. Casper-Lindley C, Yildiz FH. VpsT is a transcriptional regulator required for expression of $v p$ biosynthesis genes and the development of rugose colonial morphology in Vibrio cholerae O1 El Tor. J Bacteriol. 2004;186(5): 1574-8.

41. Utada AS, Bennett RR, Fong JCN, Gibiansky ML, Yildiz FH, Golestanian R, Wong GCL. Vibrio cholerae use pili and flagella synergistically to effect motility switching and conditional surface attachment. Nat Commun. 2014;5:4913.

42. Wadhams GH, Armitage JP. Making sense of it all: bacterial chemotaxis. Nat Rev Mol Cell Biol. 2004;5(12):1024-37.

43. Marles-Wright J, Lewis RJ. Stress responses of bacteria. Curr Opin Struct Biol. 2007;17(6):755-60.

44. Aertsen A, Michiels CW. Stress and how bacteria cope with death and survival. Crit Rev Microbiol. 2004;30(4):263-73.

45. Joelsson A, Kan B, Zhu J. Quorum sensing enhances the stress response in Vibrio cholerae. Appl Environ Microbiol. 2007;73(11):3742-6.

46. Ringgaard S, Hubbard T, Mandlik A, Davis BM, Waldor MK. RpoS and quorum sensing control expression and polar localization of Vibrio cholerae chemotaxis cluster III proteins in vitro and in vivo. Mol Microbiol. 2015;97(4): 660-75.

47. Weber B, Croxatto A, Chen C, Milton DL. RpoS induces expression of the Vibrio anguillarum quorum-sensing regulator VanT. Microbiology. 2008; 154(Pt 3):767-80.

48. Tian Y, Wang Q, Liu Q, Ma Y, Cao X, Zhang Y. Role of RpoS in stress survival, synthesis of extracellular autoinducer 2, and virulence in Vibrio alginolyticus. Arch Microbiol. 2008;190(5):585-94.

49. Hjerde E, Karlsen C, Sørum H, Parkhill J, Willassen NP, Thomson NR. Cocultivation and transcriptome sequencing of two co-existing fish pathogens Moritella viscosa and Aliivibrio wodanis. BMC Genomics. 2015;16:447.

50. Flemming HC, Neu TR, Wozniak DJ. The EPS matrix: the "house of biofilm cells". J Bacteriol. 2007;189(22):7945-7.
51. Guan J, Xiao X, Xu S, Gao F, Wang J, Wang T, Song Y, Pan J, Shen X, Wang $Y$. Roles of RpoS in Yersinia pseudotuberculosis stress survival, motility, biofilm formation and type VI secretion system expression. J Microbiol. 2015;53(9): 633-42.

52. Corona-Izquierdo FP, Membrillo-Hernandez J. A mutation in rpoS enhances biofilm formation in Escherichia coli during exponential phase of growth. FEMS Microbiol Lett. 2002;211(1):105-10.

53. Sheldon JR, Yim MS, Saliba JH, Chung WH, Wong KY, Leung KT. Role of rpoS in Escherichia coli 0157:H7 strain H32 biofilm development and survival. Appl Environ Microbiol. 2012;78(23):8331-9.

54. Adams JL, McLean RJ. Impact of rpoS deletion on Escherichia coli biofilms. Appl Environ Microbiol. 1999;65(9):4285-7.

55. Ito A, May T, Kawata K, Okabe S. Significance of rpoS during maturation of Escherichia coli biofilms. Biotechnol Bioeng. 2008;99(6):1462-71.

56. Guttenplan SB, Kearns DB. Regulation of flagellar motility during biofilm formation. FEMS Microbiol Rev. 2013;37(6):849-71.

57. Lemon KP, Higgins DE, Kolter R. Flagellar motility is critical for Listeria monocytogenes biofilm formation. J Bacteriol. 2007;189(12):4418-24.

58. O'Toole GA, Kolter R. Flagellar and twitching motility are necessary for Pseudomonas aeruginosa biofilm development. Mol Microbiol. 1998;30(2): 295-304.

59. Klausen M, Heydorn A, Ragas P, Lambertsen L, Aaes-Jørgensen A, Molin S, Tolker-Nielsen T. Biofilm formation by Pseudomonas aeruginosa wild type, flagella and type IV pili mutants. Mol Microbiol. 2003:48(6):1511-24.

60. Watnick PI, Kolter R. Steps in the development of a Vibrio cholerae El Tor biofilm. Mol Microbiol. 1999;34(3):586-95.

61. Malamud F, Torres PS, Roeschlin R, Rigano LA, Enrique R, Bonomi HR, Castagnaro AP, Marano MR, Vojnov AA. The Xanthomonas axonopodis pv. citri flagellum is required for mature biofilm and canker development Microbiology. 2011;157(Pt 3):819-29.

62. Merritt PM, Danhorn T, Fuqua C. Motility and chemotaxis in Agrobacterium tumefaciens surface attachment and biofilm formation. J Bacteriol. 2007; 189(22):8005-14.

63. Wood TK, Gonzalez Barrios AF, Herzberg M, Lee J. Motility influences biofilm architecture in Escherichia coli. Appl Microbiol Biotechnol. 2006:72(2):361-7.

64. Macnab RM. How bacteria assemble flagella. Annu Rev Microbiol. 2003;57:77-100

65. Simon $\mathrm{R}$, Priefer $U$, Puhler A. A broad host range mobilization system for in vivo genetic engineering: transposon mutagenesis in gram negative bacteria Nat. Biotech. 1983;1:784-91.

66. Messing J, Crea R, Seeburg PH. A system for shotgun DNA sequencing. Nucleic Acids Res. 1981;9(2):309-21.

67. Dunn AK, Millikan DS, Adin DM, Bose JL, Stabb EV. New rfp- and pES213derived tools for analyzing symbiotic Vibrio fischeri reveal patterns of infection and lux expression in situ. Appl Environ Microbiol. 2006;72(1):802-10

Ready to submit your research? Choose BMC and benefit from:

- fast, convenient online submission

- thorough peer review by experienced researchers in your field

- rapid publication on acceptance

- support for research data, including large and complex data types

- gold Open Access which fosters wider collaboration and increased citations

- maximum visibility for your research: over $100 \mathrm{M}$ website views per year

At BMC, research is always in progress.

Learn more biomedcentral.com/submissions 